(e-migrinter

\title{
e-Migrinter
}

$21 \mid 2020$

Famille et migrations

\section{Le rôle des réseaux familiaux dans le déploiement du flux migratoire}

Le cas des Colombiens en Espagne

\section{Celio Sierra-Paycha}

\section{OpenEdition}

\section{Journals}

\section{Édition électronique}

URL : https://journals.openedition.org/e-migrinter/2506

DOI : 10.4000/e-migrinter.2506

ISSN : 1961-9685

\section{Éditeur}

UMR 7301 - Migrinter

\section{Référence électronique}

Celio Sierra-Paycha, "Le rôle des réseaux familiaux dans le déploiement du flux migratoire », e-

Migrinter [En ligne], 21 | 2020, mis en ligne le 21 décembre 2020, consulté le 20 mai 2021. URL : http:// journals.openedition.org/e-migrinter/2506; DOI : https://doi.org/10.4000/e-migrinter.2506

Ce document a été généré automatiquement le 20 mai 2021.

Tous droits réservés 


\title{
Le rôle des réseaux familiaux dans le déploiement du flux migratoire
}

\author{
Le cas des Colombiens en Espagne
}

Celio Sierra-Paycha

1 Sur le plan des migrations internationales, la Colombie est un pays qui se distingue par une importante émigration. Celle-ci se polarise au long du vingtième siècle autour de trois pays principaux. Le Vénézuela constitue depuis les années 1930 le premier pays vers lequel les Colombiens émigrent, circulant entre les deux pays voisins au gré des cycles économiques. Les États-Unis deviennent à partir des années 1970 un nouveau pôle d'immigration colombienne. Enfin, depuis la fin des années 1990, l'Espagne se hisse au rang de troisième pays d'accueil de la population colombienne émigrée. Entre 1999 et 2007, soit moins d'une décennie, près d'un demi-million de personnes nées en Colombie se sont implantées en Espagne. Si les facteurs macro à l'origine de ce processus migratoire sont bien connus (crise économique colombienne, croissance de l'économie espagnole), nous privilégions ici au contraire une lecture "mésosociale " (Boyd, 1989 ; Robette, 2012) de cette dynamique. Cet article s'interroge ainsi sur le rôle joué par les réseaux familiaux dans le déploiement de ce flux migratoire, en prenant pour échelle d'observation les parcours migratoires et pour cadre d'étude la période 1999-2007. Les dynamiques suivant la crise de 2007-2008 - réémigration, retour, nouvelles immigrations - ne sont pas commentées ici, l'enjeu étant d'analyser spécifiquement les débuts de ce processus migratoire ${ }^{1}$.

2 Depuis la mise en évidence du rôle des réseaux familiaux dans le déploiement des flux au cours des années 1980 (Boyd, 1989), on peut avoir tendance à surestimer leur rôle. En effet, s'il est désormais reconnu que les flux migratoires sont généralement composés de migrants appartenant au même réseau, entretenant des relations interpersonnelles et ayant parfois des relations de parenté les uns avec les autres, on ne saurait pour autant réduire les flux migratoires au seul fonctionnement d'un réseau. Certains individus partent seuls, et n'ont pas recours aux membres d'un réseau familial pour migrer. Lorsqu'ils posent le pied dans le pays d'accueil, ils ne connaissent pas 
forcément de contacts sur place et sont livrés à eux-mêmes, notamment pour trouver à se loger et à s'employer.

3 Une entrée par les lieux de la résidence de la famille au moment de migrer permet de différencier plusieurs types de parcours et plusieurs étapes de développement du système migratoire. Pour les différencier, nous proposons de distinguer les parcours structurés par les lieux de résidence familiaux, des parcours «aventuriers » qui se déploient dans des espaces peu familiers du point de vue des lieux de résidence familiaux. En migrant, ces "aventuriers" produisent de la discontinuité dans les trajectoires familiales. Comment ces deux types de parcours se situent-ils socialement et chronologiquement dans l'histoire du système migratoire Colombie/Espagne ? En quoi permettent-ils de différencier différentes phases de développement du système migratoire? Le rôle de la famille se consolide-t-il au cours de la maturation du système? Qui a recours à la famille et au réseau au cours du processus migratoire?

4 Ce travail s'appuie principalement sur l'exploitation de données quantitatives espagnoles, celles d'une enquête ayant collecté en 2007 avec une grande précision les parcours de plus de 15000 migrants dont un peu plus d'un millier de personnes nées en Colombie : l'Encuesta Nacional de Inmigrantes (ENI).

5 Après avoir présenté l'approche théorique, nous évoquerons plus précisément le contexte du flux migratoire colombien en Espagne puis l'intérêt d'utiliser l'enquête ENI. Une première partie de résultats permettra de situer le rôle de la famille dans le déploiement du flux. Une seconde partie identifiera les migrants ayant le plus recours à la famille et au réseau à travers l'exemple de la recherche de leur premier emploi en Espagne.

\section{(Dis) continuité et aventure}

6 Cette analyse de la dynamique des systèmes migratoires s'inspire de deux courants théoriques. D'une part, elle s'appuie sur une approche systémique de la migration internationale (Hägerstrand, 1957; Mabogunje, 1970 ; Zlotnik, 2003) la définissant comme un «ensemble complexe d'éléments en interaction" (Mabogunje, 2013). Cette approche reconnaît son caractère multifactoriel et multiscalaire, avec la prise en compte des déterminismes macroscopiques mais aussi du rôle des réseaux migratoires et enfin de l'autonomie du migrant dans la production du flux migratoire. D'autre part, cette étude s'appuie sur une approche simmelienne de la migration en mobilisant le concept d'" aventure " (Simmel, 1911), c'est-à-dire ce qui produit de la discontinuité relativement au reste du parcours. Très utilisé dans les approches qualitatives notamment dans l'analyse de récits de migrations à la recherche des sauts, des ellipses, des trous (Leclerc-Olive, 2009) - cette notion l'est moins dans les études quantitatives, en raison de l'absence de collecte de données documentant avec précision ces parcours.

Dans le sillage tracé par l'école mésosociale de démographie (Courgeau, 1994 ; Bonvalet et Lelièvre, 2012; Dureau et Imbert, 2014), il s'agit ici de montrer qu'une analyse fine des déplacements humains à travers la lorgnette de l'enquête quantitative et d'une collecte rigoureuse des lieux avec lesquels l'individu migrant est en interaction peut s'avérer fructueuse pour appréhender les continuités et discontinuités dans son parcours. Une enquête quantitative décrivant avec précision les parcours migratoires composant les flux migratoires présente donc deux atouts: la représentativité 
statistique pour décrire extensivement le phénomène étudié et une description approfondie du processus à l'échelle individuelle.

8 La figure de l'aventurier a été particulièrement utilisée dans le champ des études migratoires francophones (De Gourcy, 2005 ; Hily, 2009 ; Bredeloup, 2014 ; StreiffFénart, 2015) et désigne un individu qui introduit de la discontinué dans son parcours lorsque des événements (la migration par exemple, mais pour G. Simmel il s'agit aussi de l'aventure amoureuse ou de l'œuvre d'art) font irruption dans le continuum de la vie. Pour l'aventurier, l'avenir n'est pas une actualisation du présent mais se nourrit du hasard et des opportunités qui se présentent à lui. Il ne faut donc pas considérer tout parcours migratoire a priori comme une aventure. L'entrée par les lieux de résidence de la famille permet éventuellement de distinguer différents degrés de continuité/ discontinuité entre la migration en Espagne et le reste du parcours. On peut en effet se demander si la migration vers l'Espagne dans le cadre d'un regroupement familial relève du même degré d'aventure qu'une migration réalisée par une personne seule sans contact sur place. Nous proposons donc (figure 1) de distinguer d'une part les parcours migratoires familiers, en jouant sur la polysémie de l'adjectif «familier ». Celui-ci qualifie à la fois un lieu préalablement connu par le migrant mais aussi un lieu de présence familiale. Ceux-ci se définissent comme des parcours se déployant au sein d'un continuum relationnel (en raison de la présence du réseau familial en origine et à destination) malgré une discontinuité spatiale (frontières). D'autre part, nous proposons l'acception suivante du parcours migratoire aventurier: parcours se déployant dans un espace non-encore familier pour le migrant, parcours discontinu dans le référentiel spatial (frontières) et relationnel (famille et réseau migratoire).

Figure $\mathrm{n}^{\circ} 1$ : Parcours migratoire familier versus parcours migratoire aventurier

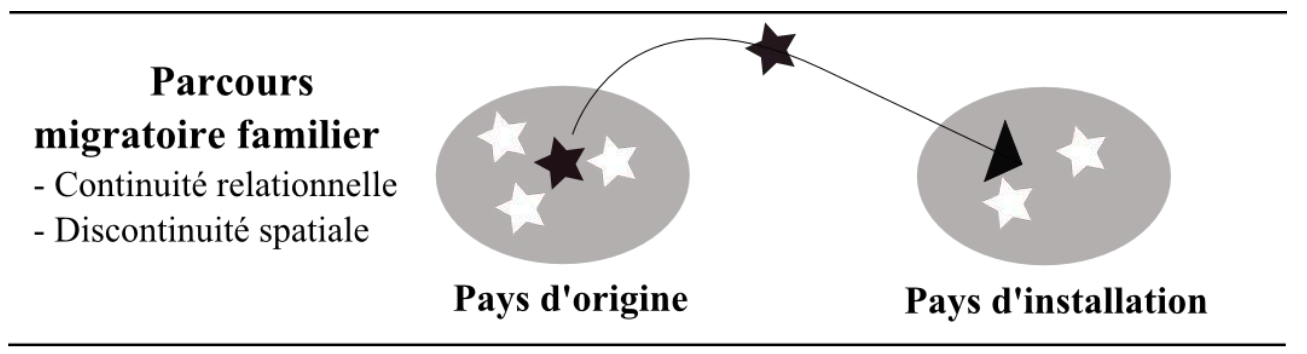

\section{Aventure}

- Discontinuité relationnelle

- Discontinuité spatiale
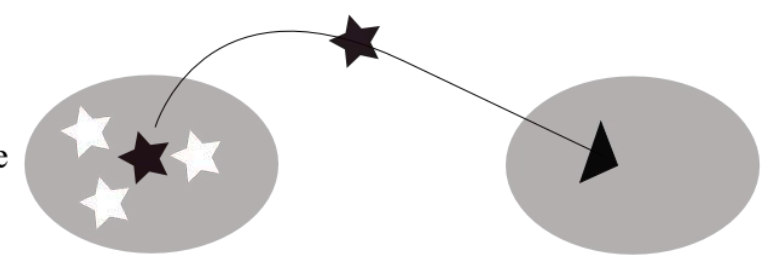

Pays d'origine

Pays d'installation

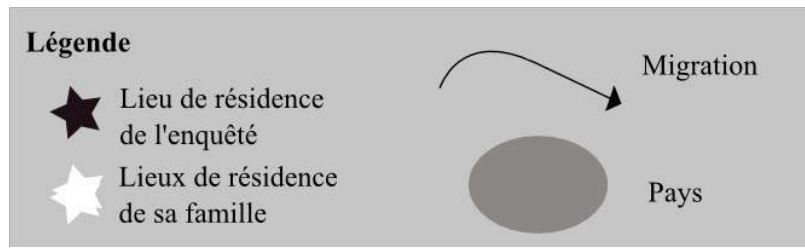

Réalisation de l'auteur. 


\section{Le déploiement du flux Colombie-Espagne}

9 Le déploiement du flux migratoire Colombie-Espagne de 1998 à 2007 est un cas intéressant pour étudier le degré de familiarité qu'entretiennent les migrants avec le pays de destination, en raison de la croissance exponentielle du flux sur cette période et de la qualité des données pour observer ce flux.

10 Avant le boom de l'émigration colombienne vers l'Espagne, la migration colombienne se faisait traditionnellement à destination du Venezuela et des États-Unis. En 1991, seulement 9343 personnes nées en Colombie vivaient en Espagne. En 2001, ce nombre s'élevait à 118148 et atteignait 379400 en 2011. L'examen des flux migratoires à partir des statistiques de changement résidentiel ${ }^{2}$ interpelle tout autant. De 1998 à 2014, la statistique publique espagnole a enregistré l'arrivée de 457000 Colombiens. Le déplacement de près d'un demi-million de personnes s'est donc produit dans un laps de temps très court, principalement entre 1998 et 2008 (figure 2). Ce phénomène devient d'autant plus intéressant à observer lorsqu'on sait que nulle institution internationale ni gouvernement n'a tenté d'en canaliser le mouvement. On peut expliquer ces variations par des déterminismes macroscopiques. Ainsi en Colombie, la crise économique et l'intensification du conflit armé ont pu jouer le rôle de facteurs d'expulsion tandis qu'en Espagne la croissance et les opportunités d'emploi peu qualifiés ont exercé une attraction sur les candidats au départ. Cette attraction a été d'autant plus forte qu'avant 2002, la loi n'exigeait pas la détention d'un visa aux ressortissants colombiens pour entrer dans l'espace Schengen. Le flux d'arrivées se réduit brutalement en 2002 avant de repartir à la hausse jusqu'à la crise de 2008, véritable turning point dans le panorama migratoire espagnol (Sierra-Paycha, 2016a).

Le système de données statistiques espagnol permet d'observer les protagonistes de ce changement. L'Encuesta Nacional de Inmigrantes (ENI) collecte des informations sur 1031 Colombiens résidant en Espagne en 2007. En divisant la population en cinq cohortes d'arrivée, on peut donc connaître les parcours des premiers migrants comme des plus récemment installés en Espagne tout en ayant au moins une centaine d'enquêtés par cohorte (figure 2). 
Figure $n^{\circ} 2$ : Flux annuels d'arrivées et de départs de Colombiens vers/depuis l'Espagne de 1988 à 2014 et définition des cohortes de Colombiens enquêtés dans l'ENI

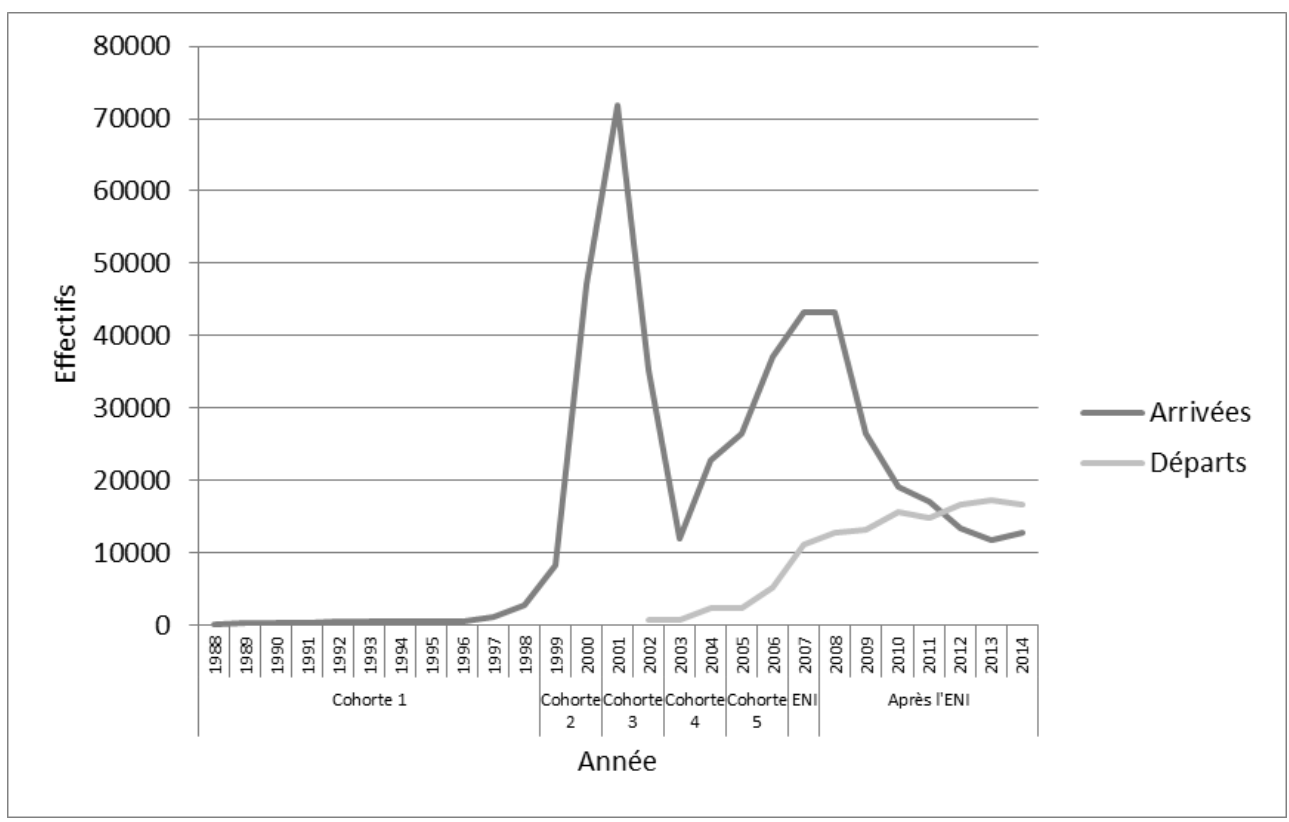

Source : INE, EVR 1988-2014.

\section{Positionner l'individu dans un réseau de liens : l'enquête ENI 2007}

L'enquête ENI a été réalisée par l'institut de statistiques espagnol en 2007 afin de mieux prendre en compte statistiquement l'immigration internationale, phénomène alors nouveau pour la péninsule ibérique. Portant sur plus de 15000 migrants (dont 1031 nés en Colombie), elle est représentative de l'ensemble de la population migrante en Espagne. Son questionnaire élaboré par des chercheurs spécialistes des migrations et de la famille ${ }^{3}$ documente avec précision le parcours migratoire en accordant une place importante à l'utilisation du réseau familial. Contrairement aux recensements et registres de population, sources statistiques classiques rattachant traditionnellement les enquêtés à deux lieux d'appartenance spatiale, à savoir le lieu de résidence actuel et le lieu de naissance, l'ENI permet de situer l'individu dans un réseau de lieux plus vaste. Le questionnaire comporte un certain nombre de questions sur le rôle de la famille dans le parcours migratoire. Une question interroge la personne sur l'éventuelle influence d'un ancien migrant en Espagne de retour dans son pays de naissance, sur la décision d'y migrer de l'enquêté. Si une personne a bel et bien exercé une telle influence, le questionnaire propose de définir le type de lien qui unit la personne enquêtée à la personne influente en trois modalités: membre de la famille, ami ou voisin, autre personne. Une autre question interroge sur l'existence d'une personnecontact en Espagne au moment de l'arrivée (alguien a quién dirigirse). En cas de réponse positive de l'enquêté, celui-ci peut alors détailler le type de lien qui l'unissait au contact en Espagne: membre de la famille, employeur ou agence d'emploi, passeur, amis, connaissances, autre type de lien.

De plus, l'ENI permet de disposer des lieux actuels et passés, de résidence de la famille nucléaire et de la fratrie, à savoir les lieux de résidence du conjoint, des parents, des 
enfants et des frères et sœurs. Nous connaissons ainsi les lieux de résidence de ces différents liens familiaux au moment de la migration vers l'Espagne. Enfin, une série de questions collecte le(s) motif(s) associé(s) à chacun des moments du parcours, que l'enquêté peut qualifier de motif pour retraite, pour mutation professionnelle, pour cause de chômage, pour recherche d'un meilleur emploi, pour des motifs religieux, pour des motifs politiques, pour raisons académiques, pour améliorer la qualité de vie, pour des raisons familiales (regroupement familial), en raison du coût de la vie, à cause du climat, pour motif de séjour temporaire en vue d'un transit vers un autre pays, pour d'autres raisons.

Grâce à ces données, il est possible d'analyser de manière précise le rôle de la famille dans le déploiement du flux migratoire Colombie-Espagne.

\section{Le rôle de la famille dans le déploiement du flux}

Le rôle de la famille a été de plus en plus important au fur et à mesure du développement $\mathrm{du}$ système migratoire colombo-espagnol ${ }^{4}$. En effet, on peut différencier les différents stades de développement du système migratoire émergence, développement - selon l'étendue des liens dont disposent les migrants qui arrivent en Espagne. Dans un premier temps, la plupart des individus migrent sans forcément avoir été influencés par une tierce personne, sans avoir de contact en Espagne et $a$ fortiori sans qu'un membre de leur famille nucléaire les précède. Dans un second temps, la tendance à la migration sans présence familière en Espagne diminue. La nature du réseau disponible en Espagne se modifie également au fil de l'arrivée du flux. Il s'agit en effet de moins en moins de liens amicaux et de plus en plus de liens familiaux en proportion.

Cette analyse $\mathrm{du}$ développement du système migratoire selon la familiarité qu'entretiennent les migrants avec la destination, montre ainsi un phénomène croissant de sélection des candidats au départ selon le critère d'une expérience familiale antérieure de migration internationale, phénomène d'autant plus important que le changement de politique migratoire espagnole en 2002 a restreint la migration colombienne au seul cadre du regroupement familial. 
Figure $n^{\circ} 3$ : Analyse de correspondances multiples sur les migrants colombiens en Espagne

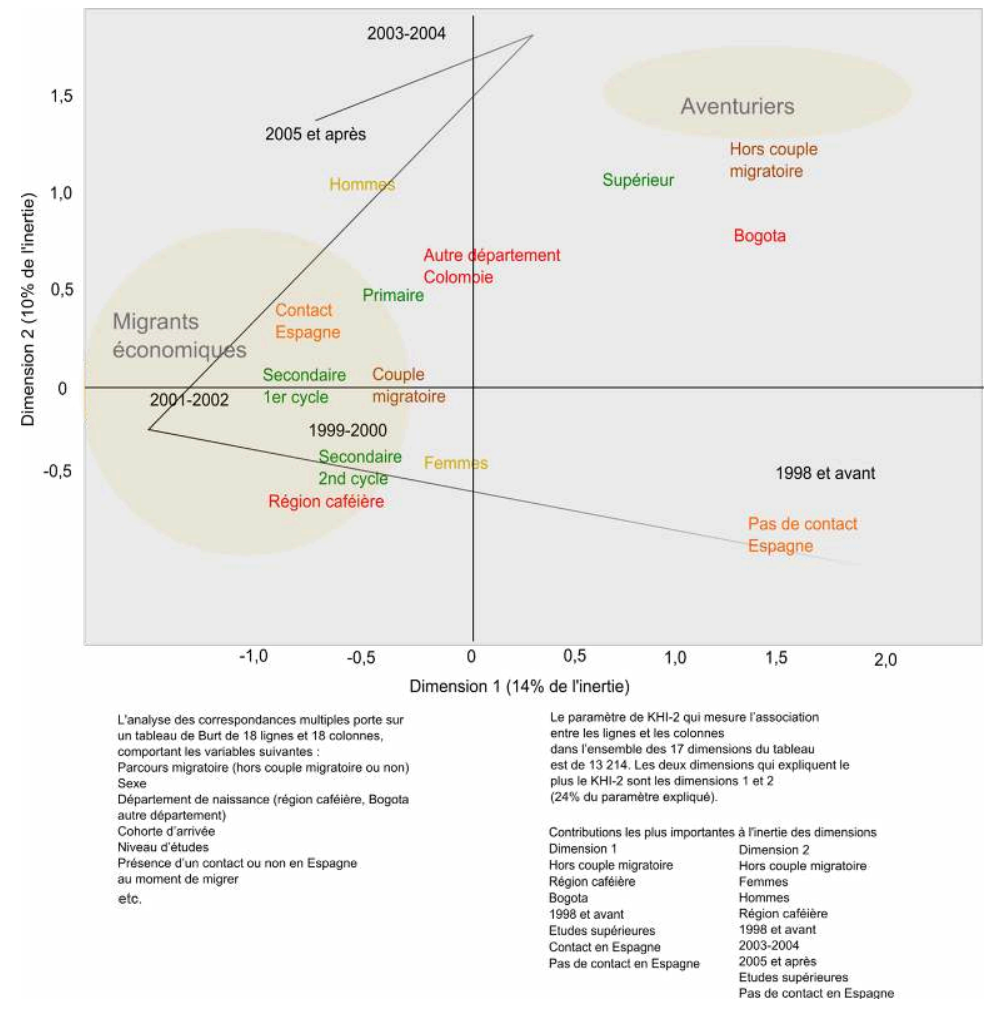

Source : INE, ENI 2007, N =1 031

17 Nous proposons ici de résumer (figure ${ }^{\circ} 3$ ) ce rôle par une analyse des correspondances multiples ${ }^{5}$ effectuée à partir d'un tableau de contingence croisant le parcours migratoire (hors couple migratoire, c'est-à-dire ayant parcouru d'autres pays avant l'arrivée en Espagne ou non), le sexe, le département de naissance (région caféière, Bogota, autre département), la cohorte d'arrivée, le niveau d'études et la présence d'un contact ou non en Espagne au moment de migrer. Le paramètre de khi-2 qui mesure l'association entre les lignes et les colonnes dans l'ensemble des 17 dimensions du tableau est de 13 214. 24\% de l'ensemble du khi-2 et de l'inertie s'expliquent grâce aux deux premières dimensions ici représentées ${ }^{6}$. Parmi les modalités qui contribuent le plus à l'inertie de la première dimension, il faut citer les parcours hors couple migratoire, les ressortissants de la région caféière et ceux de Bogota, les arrivées avant 1999, les études supérieures, le contact en Espagne et l'absence de contact en Espagne. Sur le premier axe, la dispersion de la population se structure autour d'une opposition entre départements d'origine en Colombie, qui va de pair avec une opposition selon la présence de contacts en Espagne. On a donc à gauche les personnes nées dans la région caféière et les personnes arrivées avec un contact en Espagne et à droite les personnes arrivées sans contact et plutôt issues de Bogota. Ce choix de différencier les migrants selon leur origine départementale s'explique par les attractions privilégiées exercées par certaines destinations internationales sur des régions bien définies en Colombie. Ainsi les migrants de la région caféière se sont spécialisés dans l'émigration vers l'Espagne tandis que ceux de la côte caraïbe privilégient le Venezuela comme destination alors que les Bogotains et les Antioqueños (de la région de Medellin) migrent le plus souvent aux États-Unis (figure $\mathrm{n}^{\circ} 4$ ). 

les modalités qui contribuent le plus à l'inertie, il faut mentionner les parcours hors couple migratoire (ayant transité par d'autres pays avant d'aboutir en Espagne), les femmes, les hommes, les personnes de la région caféière, les arrivées avant 1998 et les arrivées après 2003, les études supérieures, l'absence de contact en Espagne. Le deuxième axe structure donc la dispersion des Colombiens selon le sexe et selon l'âge du système migratoire. Le bas du repère est marqué par les cohortes les plus anciennement arrivées, on y trouve plutôt des femmes. Le haut du repère correspond aux cohortes arrivées les plus récemment. Les hommes y sont surreprésentés.

Une ligne reliant les cohortes entre elles par ordre chronologique d'arrivée permet de suivre le fil de l'émergence du système migratoire au sein de cet espace bidimensionnel. La réalité apparaît ici bien plus complexe qu'une progression linéaire par succession de «profils » de migrants. Sur ce plan, la véritable opposition structure d'un côté les migrants touchés par la crise colombienne, provenant surtout de la région caféière, moyennement qualifiés, ayant effectué des parcours directs depuis la Colombie. Dans le questionnaire de l'enquête, ces personnes déclarent que leur mobilité est motivée en premier lieu par la nécessité économique et dans un second temps par une volonté de regroupement familial. Aux marges du pic d'arrivée des Colombiens de la région caféière entre 1999 et 2002, on retrouve des parcours beaucoup plus diversifiés. Avant 1999, ce qui caractérise ces parcours, c'est surtout l'absence de liens familiaux et de contact sur place. Après 2003 (et donc après l'arrivée d'une centaine de milliers de Colombiens), au contraire, la probabilité d'avoir un contact en Espagne est bien plus forte. Des parcours moins caractérisés par des urgences économiques se répartissent de part et d'autre des milliers d'arrivées annuelles depuis la région caféière. Nous retrouvons d'ailleurs dans la diagonale opposée aux migrants économiques, des trajectoires mues par d'autres raisons, mais aussi les parcours hors couple migratoire, et les originaires de Bogota. La catégorie des diplômés du supérieur se situe au bout de cette diagonale. Cette information renforce donc l'hypothèse que dans le système migratoire colombien, "l'aventure» au sens d'absence de lien à destination, et de manière générale les motivations non-économiques, pourraient être situées socialement, c'est-à-dire réservées aux classes les plus aisées, et nuance l'idée d'une diffusion de cette caractéristique à tous les groupes sociaux.

Les marqueurs de l'aventure, à savoir la discontinuité relationnelle et spatiale dans le parcours, sont donc situés. Nous allons vérifier dans la suite de l'article les possibles usages du réseau familial dans l'insertion des migrants dans le pays de destination à travers l'exemple de l'insertion professionnelle.

\section{Qui a recours au réseau familial ?}

21 L'objectif de cette section est de comprendre qui a recours au réseau familial dans le processus migratoire, à travers l'exemple de l'accès au premier emploi en Espagne en analysant cela au plus près des individus et de leurs interactions.

Il est intéressant de remarquer que peu d'individus n'ont pas su répondre à la demande des enquêteurs portant sur les différentes modalités de l'accès au premier emploi ${ }^{7}$. Les enquêtés pouvaient ainsi déclarer plusieurs modes d'accès parmi la liste proposée. Malgré la possibilité de faire un choix multiple, on remarque la prépondérance de la famille et des amis parmi les réponses. $72 \%$ des Colombiens déclarent avoir obtenu ce

e-Migrinter, 21 | 2020 
premier emploi par l'intermédiaire de la famille ou d'amis. Ce mode d'accès au premier emploi arrive donc très loin devant les autres modes : $10 \%$ seulement déclarent avoir obtenu leur premier emploi par un employeur directement, $8 \%$ par la consultation d'offres d'emploi sur internet. Les autres moyens d'accès au premier emploi sont en comparaison très faibles, puisque moins de $3 \%$ des migrants y ont eu recours (par l'agence publique d'emploi, par une agence privée de recrutement, en montant sa propre affaire, par un syndicat, par des entretiens d'embauche).

23 La source principale d'informations pour obtenir un emploi se définit donc dans un périmètre restreint autour de chaque acteur. La modalité exacte en espagnol est « $a$ traves de amigos o parientes ». Dans cette modalité, les répondants peuvent englober une grande variété de liens plus ou moins forts. Parmi toutes les modalités proposées, c'est la seule source dont les contours dépendent systématiquement de chaque individu, (chaque personne a son propre cercle d'amis ou de parents, ce cercle n'existe que pour soi) contrairement aux autres modes qui se définissent indépendamment de chaque répondant (ils existent en tant qu'employeurs, agences d'emploi, offres sur internet en soi).

Qui sont les individus qui obtiennent leurs emplois au sein de ce cercle d'amis et de parents?

\section{Les moins dotées en autres ressources}

Pour analyser les caractéristiques des migrants privilégiant le réseau personnel pour se procurer un emploi, nous avons effectué une régression logistique qui modélise la probabilité d'avoir utilisé son réseau en fonction d'une batterie de variables explicatives : le sexe, le niveau d'études, la région de naissance en Colombie, la cohorte d'arrivée en Espagne et le statut juridique au moment de l'enquête.

Le choix du sexe renvoie à une littérature insistant sur le caractère différencié de l'utilisation du réseau migratoire dans l'installation selon le genre ; ainsi Boyd (1989) fait-elle l'hypothèse que les femmes migreraient plus souvent en ayant recours au réseau que les hommes. Le choix du niveau d'études est au cœur de l'hypothèse centrale des approches en termes de réseau (Tilly et Brown, 1967). On l'a vu dans d'autres publications personnelles (Sierra-Paycha, 2016c ; Sierra-Paycha, 2016b), cette variable est systématiquement fondamentale pour comprendre des différences d'usage $\mathrm{du}$ réseau personnel au cours du parcours migratoire. Les personnes faiblement dotées en capital scolaire seraient défavorisées sur le marché de l'emploi et seraient donc contraintes de compter sur les opportunités d'emploi de proximité, i.e. celles qui leur seraient accessibles par l'intermédiaire de leurs proches. Le choix du lieu d'origine renvoie à l'hypothèse de fonctionnements familiaux différents selon les régions d'origine. Le choix de la période d'arrivée permet d'identifier d'éventuels effets diachroniques sur la possibilité de s'appuyer sur le réseau pour commencer à travailler. En effet, pour les cohortes de migrants les plus anciennement installées sur le territoire espagnol (avant 1999), il est fort possible qu'elles aient par définition, moins eu d'opportunités d'emploi par le réseau, du simple fait que leur réseau ne se trouvait probablement pas en Espagne à ce moment-là, ce qui leur confère alors un statut de cohortes "pionnières ». Enfin, le choix du statut juridique permet de prendre en compte l'extrême sensibilité des opportunités d'emploi pour les migrants à la situation administrative, l'idée étant que certains secteurs d'activité fonctionneraient 
structurellement par le recrutement de main d'œuvre immigrée au statut juridique précaire. Ainsi, Emmanuel Terray et ses co-auteurs, à la suite de Michael Burawoy ontils dénoncé l'impact de ce statut précaire, sur le versement de salaires au rabais dans les secteurs d'activité non-délocalisables tels le tourisme, la restauration, le bâtiment, les services à la personne (Burawoy, 1976 ; Balibar et al., 1999).

Les résultats de la régression montrent que la détention d'un capital scolaire important semble entraîner un moindre recours au réseau tandis que les individus peu diplômés y ont davantage recours. Bien que les résultats ne soient pas statistiquement significatifs concernant le titre de séjour, on observe tout de même un gradient négatif du recours au réseau selon la précarité juridique. Ainsi, les personnes naturalisées, sont celles qui ont le moins recours au réseau ; viennent ensuite les étrangers en situation régulière, et enfin les étrangers en situation irrégulière. En revanche, les résultats de la régression logistique ne permettent pas d'accepter l'hypothèse d'un recours différencié au réseau personnel pour trouver un emploi selon le sexe.

Ces résultats rejoignent les conclusions de Charles Tilly et Harold Brown qui dans un article précurseur sur le rôle des réseaux personnels dans la migration, formulaient l'hypothèse suivante: le recours aux réseaux personnels pour se procurer un emploi varie en fonction d'autres capitaux que peuvent faire valoir les migrants sur le marché du travail (Tilly et Brown, 1967). Ainsi, selon ce modèle, les moins qualifiés, les moins dotés socio économiquement, les moins expérimentés, compteraient davantage sur leurs proches, que sur leurs ressources propres (diplôme, curriculum vitae, maîtrise de la langue usitée sur le marché du travail) pour obtenir un emploi. La littérature sur les migrations colombiennes affirme également que ce sont les personnes les moins qualifiées qui utilisent leurs liens faibles pour chercher un emploi (Gincel, 2007; Guarnizo, 2008 ; Magnan Penuela, 2009). 
Tableau $n^{\circ} 1$ : Régression logistique sur le fait d'avoir eu recours au réseau proche pour se procurer un emploi

\begin{tabular}{|c|c|c|c|}
\hline $\begin{array}{l}\text { Ref=ne pas avoir trouvé un } \\
l^{10} \text { emploi grâce au réseau }\end{array}$ & Odds-ratio & $\begin{array}{l}\text { Intervalle d } \\
95 \%\end{array}$ & de Wald à \\
\hline $\begin{array}{l}\text { Sere } \\
\text { Femmes }\end{array}$ & $\begin{array}{l}\text { Ref. } \\
1,000\end{array}$ & $\begin{array}{l}\text { Ref. } \\
0,729\end{array}$ & $\begin{array}{l}\text { Ref. } \\
1,373\end{array}$ \\
\hline $\begin{array}{l}\text { Niveau d'études } \\
\text { aucun diplôme ou études } \\
\text { primaires } \\
\text { secondaire ler cycle } \\
\text { secondaire 2nd cycle } \\
\text { supérietrr }\end{array}$ & $\begin{array}{l}2,458^{*} \\
2,161 \\
1,845 \\
\text { Ref. }\end{array}$ & $\begin{array}{l}1,510 \\
1,258 \\
1,256 \\
\text { Ref. }\end{array}$ & $\begin{array}{l}4,001 \\
3,712 \\
2,710 \\
\text { Ref. }\end{array}$ \\
\hline $\begin{array}{l}\text { Région de naissance en } \\
\text { Colombie } \\
\text { Région caféière } \\
\text { Autre }\end{array}$ & $\begin{array}{l}\text { Ref. } \\
1,928^{* * * *} \\
1,245\end{array}$ & $\begin{array}{l}\text { Ref. } \\
1,175 \\
0,763 \\
\end{array}$ & $\begin{array}{l}\text { Ref. } \\
3,164 \\
2,031 \\
\end{array}$ \\
\hline $\begin{array}{l}\text { Cohorte d'arrivéc } \\
\text { l998 el avant } \\
1999-2000 \\
2001-2002 \\
2003-2004 \\
2005-2006\end{array}$ & $\begin{array}{l}\text { Rcf. } \\
0,938 \\
1,091 \\
0,789 \\
1,285\end{array}$ & $\begin{array}{l}\text { Rcf. } \\
0,593 \\
0,672 \\
0,402 \\
0,619 \\
\end{array}$ & $\begin{array}{l}\text { Ref. } \\
1,483 \\
1,770 \\
1,549 \\
2,671\end{array}$ \\
\hline $\begin{array}{l}\text { Statut juridique } \\
\text { Espagnole } \\
\text { UE29 } \\
\text { Régulière } \\
\text { Irrégulière }\end{array}$ & $\begin{array}{l}\text { Ref. } \\
0,927 \\
1,457 \\
2,310\end{array}$ & $\begin{array}{l}\text { Ref. } \\
0,047 \\
0,965 \\
0,998\end{array}$ & $\begin{array}{l}\text { Ref. } \\
18,320 \\
2,198 \\
5,343\end{array}$ \\
\hline
\end{tabular}

Champ : les migrants colombiens ayant eu un emploi en Espagne et déclaré un mode d'accès au premier emploi. $\mathrm{N}=931$ personnes,

Note de lecture : Un odds-ratio supérieur à 1 indique un effet positif de la variable étudiée, un oddsratio inférieur à 1 indique un effet négatif. Les odds-ratios significatifs au seuil de $10 \%$ ont été annotés d'un astérisque dans le tableau $(* \star *:<0,001 ; * *:<0,01 ; *:<0,1)$. Pour chaque variable, la modalité de référence choisie dans le modèle est indiquée en italique. Par exemple, toutes choses égales par ailleurs, la probabilité d'avoir trouvé le premier emploi grâce à la famille et aux amis est supérieure pour les individus nés dans la région caféière relativement aux personnes nées à Bogota.

Source : INE, ENI, 2007. Réalisation de l'auteur.

\section{Certaines origines régionales fonctionnent en réseau migratoire}

Ainsi, la probabilité du recours au réseau diminue selon le niveau d'études tandis qu'elle augmente avec le niveau de précarité juridique (même si ce dernier résultat n'est pas ici significativement dissocié des autres variables explicatives). L'intérêt d'analyser ce processus à l'échelle infranationale est à nouveau confirmé car l'on remarque que les personnes issues de la région caféière (à savoir les départements Valle del Cauca, Risaralda et Quindío) ont deux fois plus de chances de recourir au réseau que celles provenant de Bogota. Rappelons ici que cette région est spécialisée dans l'envoi de migrants colombiens vers l'Espagne. La surreprésentation des personnes nées dans la région caféière parmi celles qui utilisent le réseau rappelle l'importance du recours à la famille élargie pour se loger pour cette même population. On a en effet montré que les personnes originaires de la région de Cali connaissaient un risque de corésider avec de la famille élargie plutôt qu'avec la famille nucléaire, près de deux fois supérieur à celui des Bogotains (Sierra-Paycha, 2016b). 
Figure $n^{\circ} 4$ : Le déploiement international de la migration colombienne par origines régionales

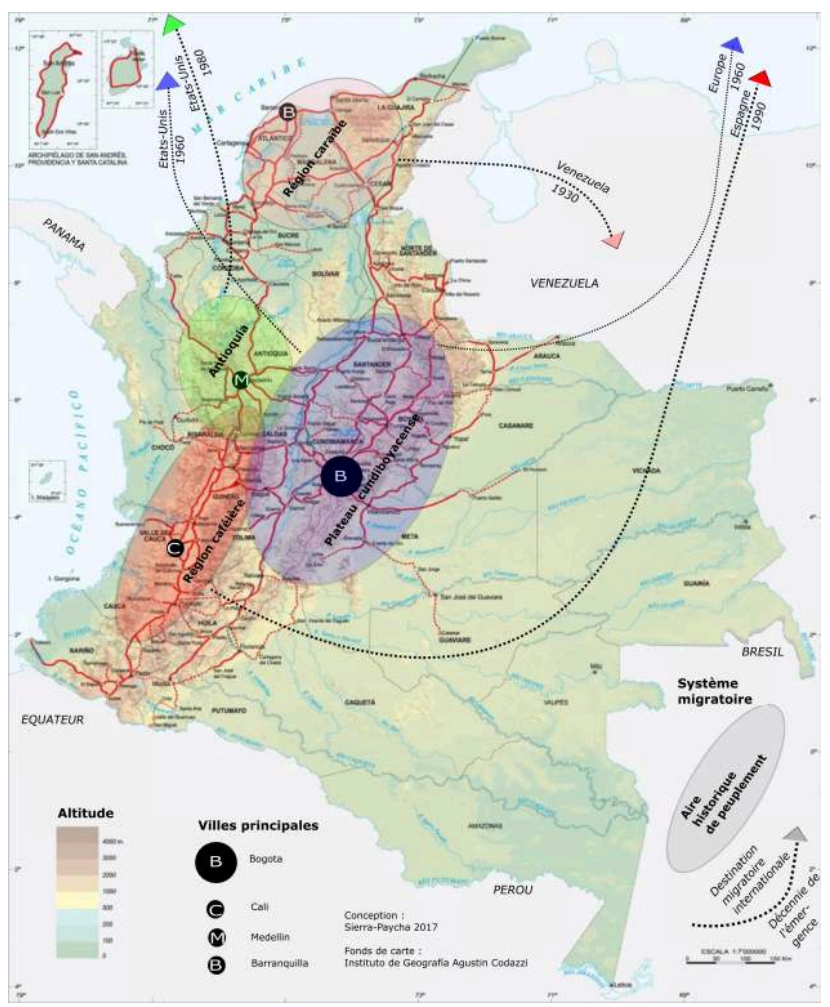

Conception : Sierra-Paycha, 2017. Fonds de carte : Instituto de Geografía Augustin Codazzi.

30 Cette analyse du recours au réseau selon l'origine régionale des migrants montre donc que les migrants inscrits dans des réseaux migratoires structurés comme celui de la région caféière pour les Colombiens ont tendance à effectuer des parcours internationaux familiers et non aventuriers. L'aventure concerne davantage les migrants dont les réseaux familiaux et amicaux ne sont pas ancrés dans le pays d'accueil.

\section{Conclusion}

Une entrée par le réseau familial fournit un bon indicateur pour apprécier la maturation d'un système migratoire : moins sollicité aux prémices, il l'est de plus en plus au cours de la consolidation du système. On assiste en effet à une sélection progressive des nouveaux migrants par la présence antérieure de la famille en Espagne. Si l'on considère à l'instar de Simmel que l'aventure consiste à introduire de la discontinuité dans son parcours, on remarque donc que l'émergence d'un système migratoire dans un nouveau pays d'accueil repose sur l'accumulation d'un certain nombre de parcours migratoires aventuriers, c'est-à-dire de personnes qui migrent vers l'inconnu, dans un lieu qui n'appartient pas à un espace de vie familier comme pourrait l'être une migration dans une destination où le réseau est déjà en place.

L'accumulation de parcours migratoires aventuriers depuis la région caféière en Colombie dans les années 1990, aux prémices du système migratoire Colombie-Espagne, a permis dans un second temps à leurs proches de les rejoindre dans les années 2000 lorsque l'afflux de Colombiens est devenu massif. En effet, la connaissance du pays 
d'accueil, en l'occurrence l'Espagne, à travers la présence du réseau familial permet de pallier une moindre dotation en autres ressources (diplôme, statut juridique) et une politique migratoire de plus en plus restrictive (grâce aux demandes de regroupement familial). Le paradigme de la migration aventurière ne s'applique qu'à la minorité d'individus les plus qualifiés et cumulant une importante expérience migratoire préalable.

Le rôle du réseau familial est donc fondamental dans le déploiement massif de flux migratoire grâce à sa capacité à rendre familier le lieu de destination pour les candidats au départ issues d'une région déterminée. L'utilisation répétée des réseaux familiaux pour migrer, se loger, trouver un emploi dans le pays d'accueil permet de lier les facteurs d'expulsion dans le pays d'origine (crise économique en Colombie) et les facteurs d'attraction dans le pays de destination (boom économique en Espagne). Par ce mécanisme, les réseaux familiaux génèrent in fine des systèmes migratoires structurés connectant deux lieux (la région caféière en Colombie et l'Espagne) malgré la discontinuité spatiale (et juridique) qui les sépare.

\section{BIBLIOGRAPHIE}

Baccini, Alain (2010) Statistique Descriptive Multidimensionnelle, Toulouse, Institut de Mathématiques de Toulouse, $33 \mathrm{p}$.

Balibar, Etienne ; Chemillier-Gendreau, Monique ; Costa-Lascoux, Jacqueline ; Terray, Emmanuel (1999) Sans-papiers : l'archaïsme fatal, Paris, La Découverte,128 p. (Sur le vif).

Benzecri, Jean-Paul (1973) L'Analyse des données. Tome 2 :l'analyse des correspondances, Paris, Dunod, $619 \mathrm{p}$.

Bonvalet, Catherine ; Lelièvre, Eva (2012) De la famille à l'entourage : l'enquête Biographies et entourage, Paris, Ined éditions, 470 p. (Grandes enquêtes).

Boyd, Monica (1989) Family and Personal Networks in International Migration : Recent Developments and New Agendas, International Migration Review, vol. 23, n 3, pp. 638-670.

Bredeloup, Sylvie (2014) Migrations d'aventures. Terrains africains, Paris, Éditions du Comité des Travaux Historiques et Scientifiques, 141 p. (Géographie).

Burawoy, Michael (1976) The functions and reproduction of migrant labor : comparative material from Southern Africa and the United States, American Journal of Sociology, vol. 81, $\mathrm{n}^{\circ} 5$, pp. 1050-1087.

Courgeau, Daniel (1994) Du groupe à l'individu : l'exemple des comportements migratoires, Population, vol. 49, $\mathrm{n}^{\circ} 1$, pp. 7-26.

De Gourcy, Constance (2005) L'autonomie dans la migration. Réflexion autour d'une énigme, Paris, L'Harmattan, 347 p. (Logiques sociales).

Dureau, Françoise ; Imbert, Christophe (2014) L'approche biographique des mobilités résidentielles, In Imbert, C. ; Dubucs, H. ; Dureau, F. ; Giroud, M. (dir.) D'une métropole à l'autre : 
pratiques urbaines et circulations dans l'espace urbain européen, Paris, Armand Colin, pp. 33-80, (Recherches).

Gincel, Anne (2007) Un village colombien à Paris, Hommes \& migrations, $\mathrm{n}^{\circ}$ 1270, pp. 32-42.

Guarnizo, Luis Eduardo (2008) Londres latina. La presencia colombiana en la capital británica, Zacatecas, Universidad Autónoma de Zacatecas/Miguel Angel Porrua, 178 p.

Hägerstrand, Torsten (1957) Migration and area. Survey of a sample of Swedish migration fields and hypothetical considerations on their genesis, In Hannerberg, D. ; Hägerstrand, T. ; Odevings, B. (dir.) Migration in Sweden. A symposium, Lund, Lund studies in Geography, pp. 27-158 (série B).

Hily, Marie-Antoinette (2009) L'usage de la notion de "circulation migratoire" In Cortes, G. ; Faret, L. (dir.) Les circulations transnationales. Lire les turbulences migratoires contemporaines, Paris, Armand Colin, pp. 23-28 (U Sciences humaines et sociales).

Leclerc-Olive, Michèle (2009) Enquêtes biographiques entre bifurcations et événements. Quelques réflexions épistémologiques, In Grossetti, M. ; Bessin, M. ; Bidart, C. (dir.) Bifurcations, Paris, La Découverte, pp. 329-346 (coll. Recherches).

Le Petitcorps, Colette (2015) Service à domicile, femmes et migrations en France. Le rapport de domesticité en question, Poitiers, Université de Poitiers, 341 p.,

Th. Doct : Socio. : Poitiers : 2015.

Mabogunje, Akin (2013) Une approche systémique de la théorie de la migration rurale-urbaine In Piché, V. (dir.) Les théories de la migration, Paris, Ined éditons, pp. 151-161 (Textes fondamentaux).

Mabogunje, Akin (1970) Systems Approach to a Theory of Rural-Urban Migration, Geographical Analysis, vol. 2, n 1, pp. 1-18.

Magnan Penuela, Marion (2009) Migration, réseaux transnationaux et identités locales : le cas des Colombiens à New York, Paris, Université Paris 3 Sorbonne-Nouvelle, 411 p., Th. Doct : Socio. : Paris : 2009.

Robette, Nicolas (2012) Les espaces de vie individuels : de la géographie à une application empirique en démographie, Cybergeo : European Journal of Geography, rubrique Espace Société territoire, $n^{\circ} 605$ [Disponible sur Internet].

Sierra-Paycha, Celio (2018) La famille à distance, ressource spatiale pour migrer et support de flux transnationaux, In Imbert, C. ; Lelièvre, É. ; Lessault, D. (dir.) La famille à distance, Paris, Ined éditions, pp. 93-114 (Questions de Populations).

Sierra-Paycha, Celio (2017) Morphogenèse d'un système migratoire. Emergence(s), développement(s) et transformation(s) du système migratoire Colombie/Espagne (1988-2014), Poitiers, Université de Poitiers, $388 \mathrm{p}$.,

Th. Doct : Géogr. : Poitiers : 2017.

Sierra-Paycha, Celio (2016a) Les dynamiques du champ migratoire colombo-espagnol en temps de crise, Autrepart, $\mathrm{n}^{\circ} 77$, pp. 143-164.

Sierra-Paycha, Celio (2016b) Le rôle des liens faibles dans l'insertion résidentielle des Colombiens en Espagne (2007) : une approche par le groupe de corésidents, In Cailly, L. ; Dureau, F. (dir.) Les espaces du logement : pratiques habitantes et politiques publiques, Paris, L'Harmattan, pp. 101-115 (Habitat et sociétés).

Sierra-Paycha, Celio (2016c) La circulation au sein de l'espace résidentiel de la famille. Le cas des familles de migrants colombiens en Espagne, Revue européenne des migrations internationales, vol. 32, $\mathrm{n}^{\circ}$ 1, pp. 183-209. 
Simmel, Georg (1911) La philosophie de l'aventure, Paris, L'Arche, 120 p. (Tête à tête).

Streiff-Fénart, Jocelyne (2015) Circulation, retour, transit, aventure, voyage. Les concepts des sciences sociales à l'épreuve des catégories politiques et "emic" de la mobilité, La circulation et le retour à l'épreuve des contraintes migratoires ? Journée d'études CIST/URMIS/MIGRINTER 25 septembre 2015, Paris.

Tilly, Charles ; Brown, C. Harold (1967) On uprooting, kinship and the Auspices of Migration, International Journal of Comparative Sociology, vol. 8, pp. 139-164.

Zlotnik, Hania (2003) Théories sur les migrations internationales, In Caselli, G. ; Vallin, J. ; Wunsch, G. (dir.) Démographie : analyse et synthèses IV Les déterminants de la migration, Paris, Ined éditions, pp. 55-78.

\section{NOTES}

1. Ces dynamiques postérieures à la crise ont fait l'objet d'autres publications (Sierra-Paycha, 2016a ; Sierra-Paycha, 2017)

2. Il s'agit des changements observés dans les registres de population municipaux (voir SierraPaycha, 2017, pp. 53-56 pour une description détaillée de la source).

3. Comme David Reher et Miguel Requena.

4. Cette analyse s'appuie sur les résultats d'une recherche doctorale (Sierra-Paycha, 2017) ayant déjà donné lieu à plusieurs publications (Sierra-Paycha, 2016b ; Sierra-Paycha, 2016c ; SierraPaycha, 2018) concernant le rôle de la famille en migration.

5. Il s'agit d'une méthode d'analyse descriptive multidimensionnelle (Benzecri 1973).

6. Étant donné la taille du tableau de Burt étudié (17 dimensions), un quart de la dispersion expliqué par les deux premiers axes est un résultat satisfaisant (Baccini, 2010).

7. On peut a priori être sceptique quant à la capacité des individus à déterminer le mode d'accès au premier emploi. Le processus est dans certains cas irréductible à une variable dichotomique, celui-ci est diffus dans le temps et mobilise parfois plusieurs interactions avec une variété d'acteurs. Néanmoins on remarque rétrospectivement que les individus sont la plupart du temps capables de déterminer quelle personne a le plus contribué à leur insertion professionnelle.

\section{RÉSUMÉS}

La théorie classique des migrations explique l'apparition des flux migratoires internationaux par des facteurs d'expulsion dans le pays d'origine et des facteurs d'attraction dans le pays d'accueil. La théorie mésosociale considère au contraire que les flux migratoires sont composés de personnes appartenant au même réseau d'interconnaissance. Aucune de ces deux propositions n'est pleinement satisfaisante. À partir de données quantitatives, cet article permet de faire la synthèse entre les deux approches en s'intéressant au rôle joué par la famille dans les parcours migratoires qui composent le flux d'émigration de la Colombie vers l'Espagne de 1990 à 2007. Moins sollicité aux prémices du système migratoire, le réseau familial l'est de plus en plus au cours de la consolidation du système. Le rôle du réseau est donc crucial pour amplifier un flux migratoire naissant mais il est secondaire dans la mise en place du flux et dans les parcours des 
pionniers. Quelle que soit la période d'arrivée, ce sont les individus les moins dotés en autres ressources (économiques, scolaires, juridiques) qui ont le plus recours au réseau familial pour émigrer, se loger, trouver un emploi.

INDEX

Mots-clés : flux migratoire, réseau, famille, parcours migratoire

Index géographique : Colombie, Espagne

\section{AUTEUR}

\section{CELIO SIERRA-PAYCHA}

Maître de Conférences à l'Université Paris 1 Panthéon-Sorbonne. Rattaché au Centre de Recherches de l'Institut de Démographie de Paris 1 (CRIDUP) - Chercheur associé à l'Institut National d'Études Démographiques (INED), fellow de l'Institut Convergence Migrations celio.sierra-paycha@univ-paris1.fr 